\title{
Deformation Monitoring of a Tracking Particle Detector using Fiber Bragg Grating sensors
}

\author{
M. Della Pietra* ${ }^{*}{ }^{a}$ S. Campopiano ${ }^{a b}$ and A. ladicicco ${ }^{a b}$ \\ ${ }^{a}$ Engineering Dept, University of Naples "Parthenope" \\ Centro Direzionale, Isola C4, 80143, Napoli, Italy \\ ${ }^{b}$ Istituto Nazionale di Fisica Nucleare, INFN, Sezione di Napoli \\ Via Cinthia, 80126 Napoli, Italy \\ E-mail: massimo.dellapietraduniparthenope.it
}

\begin{abstract}
In this work Fiber Bragg Grating (FBG) technology is proposed as suitable sensing solution for the real-time deformation monitoring of a micro pattern gaseous detector. The basic idea relies at with the development of a system where several FBG strain sensors are surface attached to a detector support panel. Measuring strain on both sides of the panel it is possible to obtain its local curvature that is related to the second derivatives of the shape described by the bent surface. As a demonstrative target, some FBG sensors have been integrated with a miniature detector support panel in order to investigate their potentialities/capabilities in detecting local strain and thus bending. Preliminary experimental results are presented and discussed.
\end{abstract}

Technology and Instrumentation in Particle Physics 2014

2-6 June, 2014

Amsterdam, the Netherlands

\footnotetext{
* Speaker.
} 


\section{Introduction}

The frontier evolution of a gaseous tracking particle detector technology has been moved in developing Micro-Pattern Gas Detectors that can achieve unprecedented spatial resolution, high rate capability and large sensitive area. However, also the geometry of such kind of tracking detector, in spite of the particular technology used, has to be known with a precision of the order of few tens of microns. The relative position of each detector with respect to a reference system is usually measured through optical sensor elements (CCDs, lenses, light sources), but also any deformation (bending, strain, torsion) with respect to its ideal shape has to be monitored as well. From this consideration, a real-time sensing technology capable to detect any curvature and deformation of a detector with a resolution of few tens of microns is desirable. However any sensor to be integrated with the detector itself should ideally comply with many requirements in terms of radiation hardness, insensitivity to magnetic field, and so on. Fiber Bragg gratings [1] (FBGs) seem to provide a suited solution. In fact, the fiber itself can tolerate very high levels of radiation [2], is insensitive to magnetic field and electromagnetic noise, and FBGs are intrinsic strain and temperature sensors with wavelength-encoded information, offer high multiplexing capability with reduction of cabling complexity and linear output.

\section{Methodology}

According to classical beam theory and assuming small deformation with respect to the linear dimension of a panel, its deflection can be evaluated by measuring longitudinal strain. Indeed a second derivative relation exist between the displacement orthogonal to the surface and the strain component parallel to it [3]. In figure 2 a schematic view of a solid unidimensional beam is reported. In this simplified schema, the beam (along $\mathrm{x}$ axis) is bent under a mechanical torque $\mathrm{M}$, which is along $\mathrm{y}$ axis: the beam has a deflection along $\mathrm{z}$ axis, bending with a radius of curvature $\mathrm{R}$. The bending induces compressive strain on the "inside" surface of the beam (light blue coloured) and tensile strain on the "outside" surface (red coloured): in the middle, a surface is still unstrained and we will refer to it as "neutral axis". Introducing the bending angle $\theta=1 / \mathrm{R}$ and the curvature $\kappa=1 / \mathrm{R}$, (see fig 2), the surface strain component along $\mathrm{x} \varepsilon(x)$ can be written as a function of the curvature radius; the latter is proportional to the second derivative of the displacement along $\mathrm{z}$ axis $\omega(x)$ :

$$
\varepsilon(x)=-d_{1} \frac{\partial^{2} \omega(x)}{\partial x^{2}}
$$

where $d_{1}$ is the distance of the strained surface from the neutral axis. Following this idea, if the strain profile along the beam is measured in some points, the function $\varepsilon(x)$ can be fitted with a polynomial $P_{n}(x)$ of degree $\mathrm{n}$ in order to achieve a continuous function for the strain. To compute the panel displacement we can integrate eq. 2.1 twice with respect to $\mathrm{x}$ :

$$
\omega(x)=-d_{1} \int\left(\int P_{n}(x) d x\right) d x=P_{n+2}(x)+C_{1} x+C_{0}
$$

The two integrating constants $C_{1}$ and $C_{0}$ can be calculated using appropriate boundary conditions including considerations about eventually structural symmetry, or known displacement in a specific 

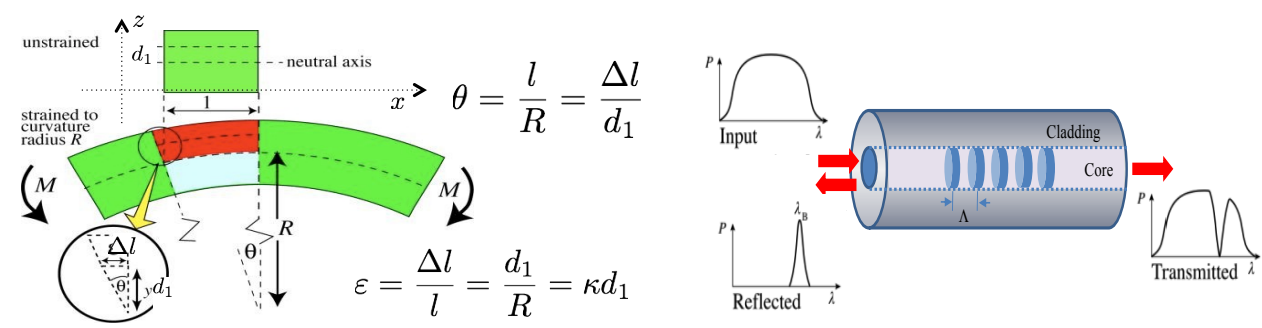

Figure 1: On the left: schematic view of beam deflection. On the right: schema of FBG sensor.

point, or the continuity of deflection and/or its first derivative. Actually $C_{0}$ acts as offset and it has no meaning when it is necessary to detect the deflection changes.

To measure the longitudinal strain component we propose to use FBG sensors. They consists of a segment of optical fiber in which a periodic modulation of the core refractive index have been induced. The primary structure of FBG is shown in fig. 2 where the typical length is about 2-20 $\mathrm{mm}$. When broadband light passes through the FBG, a narrowband spectral component at the Bragg wavelength is reflected by the FBG. The so called Bragg wavelength, $\lambda_{B}$ is given by the following expression: $\lambda_{B}=2 n_{e f f} \Lambda$, where $\lambda_{B}$ is the Bragg wavelength; $n_{\text {eff }}$ is the effective refraction index of the guided core mode; and $\Lambda$ is the periodicity of the index variation of the grating (grating pitch). The grating region can be used as sensing elements. In fact any perturbation that can change effective index $\left(n_{e f f}\right)$ and periodicity $(\Lambda)$ will result in a shift in Bragg wavelength. With more details, the Bragg grating wavelength shifts caused by temperature changes $\Delta T$ and/or local strain $\varepsilon$ can be expressed according to the following equation:

$$
\frac{\Delta \lambda_{B}}{\lambda_{B}}=S_{\varepsilon} \varepsilon+S_{T} \Delta T
$$

where $\Delta \lambda_{B}$ is the variation in Bragg wavelength, and $S_{\mathcal{E}}$ and $S_{T}$ are the sensitivity coefficients to strain and temperature, respectively. According with equation (2.3) FBG sensors permit accurate measurement of the strain profile of the support panel surfaces where some FBGs are properly bonded to (or embedded in) whereas thermal effects can be compensated by using an additional, not bonded, FBG sensor [1,2].

\section{Experimental results}

In order to test the proposed approach, in this paper we have monitored the deformation of a miniaturized support panel, that has been considered as a beam-like structure with supports at its two ends. The miniature panel sizes are of $\mathrm{L}_{2}=20 \mathrm{~cm}$ and $\mathrm{L}_{1}=40 \mathrm{~cm}$ whereas the thickness of $\mathrm{d}=9.0 \mathrm{~mm}+/-0.1 \mathrm{~mm}$ and it is formed by an honeycomb flame-resistant meta-aramid material (NOMEX) core with two skins of a composite material made of woven fiberglass cloth with an epoxy resin binder (FR4). One side of the panel has a thin copper film glued, in order to use it as an electrode inside the detector (see fig. 3b). In following it is simply modeled as a two-layer panel involving a composite top layer and a copper film. Five FBG sensors, with different coating 

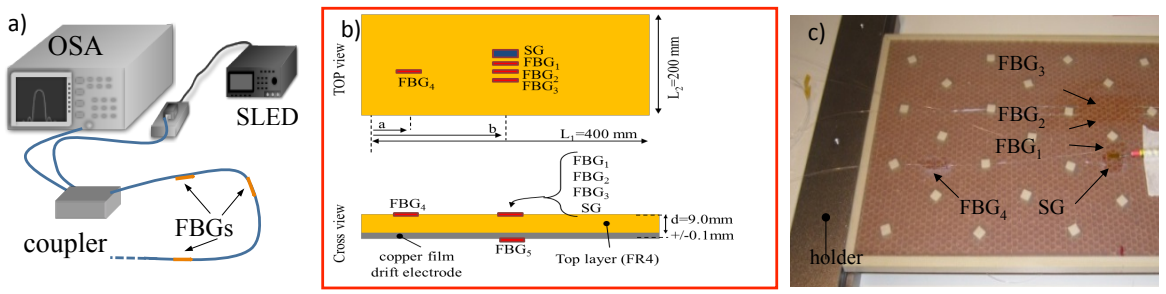

Figure 2: a) Experimental setup. b) Schematic of the panel with the position of the sensors. c) Picture of the panel.

have been glued to the panel with different glue, in two position. The aim is to compare, in terms of measured strain, the bare FBGs with protective polyamide coated FBGs since the former is easy to fix ensuring maximum strain transfer whereas the last one is more robust in handle operations. Besides we have compared different adhesives such as Araldite 2011 and a Cyanoacrylate-base adhesive in order to validate the strain transfer capability of the former one that is already certificate for operation in high radiation environment. Summarizing, in our setup five FBGs with different $\lambda_{B}$ are attached to the panel. Three of them, $\mathrm{FBG}_{1} \mathrm{FBG}_{2}$ and $\mathrm{FBG}_{3}$, with different coatings are fixed (very close each other) in the center of the top side of the support (see fig. 1(b)) with different adhesives. The $\mathrm{FBG}_{4}$ is fixed at a distance of $\mathrm{a}=6.2 \mathrm{~cm}$ from left side and the $\mathrm{FBG}_{5}$ is fixed on the back side (copper film) in specular position with respect to $\mathrm{FBG}_{1}$. Another sensor has been added to measure the temperature $\left(\mathrm{FBG}_{T}\right)$. Moreover the responses of FBGs are compared with a conventional resistive strain gauges (SG) fixed close to $\mathrm{FBG}_{1}$. The Bragg wavelength shifts in FBGs reflectance spectra are detected through an optoelectronic setup involving a $3 \mathrm{~dB}$ coupler placed between a broadband light source (Superluminescent LED) and the fiber with FBGs serially connected where one return end is sent to optical spectrum analyzer (Yokogawa AQ6317B), as schematically shown in fig. 3a. SG measurements were carried out by means of an standard Digital Multimeter. FBG strain sensors have been previously characterized in terms of their strain and temperature sensitivities exhibiting $S_{T}=5.4 \cdot 10^{-6} \mathrm{~K}^{-1}$ and $S_{\varepsilon}=0.815 \cdot 10^{-6} \mu \varepsilon^{-1}$, and thus strain measurements with resolution lower than $1 \mu \varepsilon$ are possible [1]. The Bragg wavelengths are retrieved via centroid analysis of the reflected spectra. The small temperature changes have been compensated by using $\mathrm{FBG}_{T}$. The detector panel is positioned on two rigid holders while several masses (named $\mathrm{W}_{0}=0 \mathrm{~g}, \mathrm{~W}_{1}=269 \mathrm{~g} ; \mathrm{W}_{2}=537 \mathrm{~g} ; \mathrm{W}_{3}=800 \mathrm{~g} ; \mathrm{W}_{4}=1432 \mathrm{~g}$, respectively) are applied in different points along the panel length, to achieve mechanical deformation of the panel itself. The fig. 3a plots the strain measurements of optical and electrical sensors versus the time (with scan step of 15 seconds) during a stepwise weight increment (load) and decrement (unload), applied in the middle of the panel. From these data some important results can be highlighted. $\mathrm{FBG}_{1}, \mathrm{FBG}_{2}, \mathrm{FBG}_{3}$ and $\mathrm{FBG}_{4}$ record a negative strain while $\mathrm{FBG}_{5}$ records a positive strain. This means that , as expected, the top layer surface is compressed while the opposite surface (bottom copper layer) is stretched. Comparing $\mathrm{FBG}_{1}$ and $\mathrm{FBG}_{5}$ measurements the position of the neutral axis can be easily derived with a liner interpolation. Also, it can be noted that responses of $\mathrm{FBG}_{1}, \mathrm{FBG}_{2}, \mathrm{FBG}_{3}$ and $\mathrm{SG}$ are in perfect agreement (within $+/-1 \mu \varepsilon$ ). This means that bare and polyamide FBGs are both well candidate for our purpose and Araldite 2011 permits a full strain 
a)

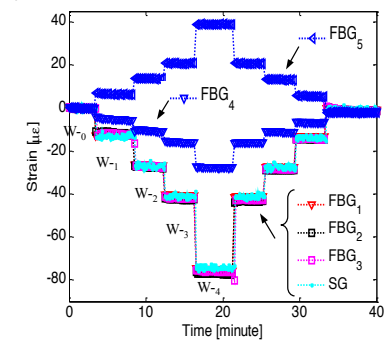

b)

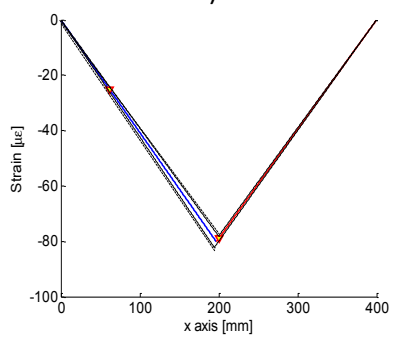

c)

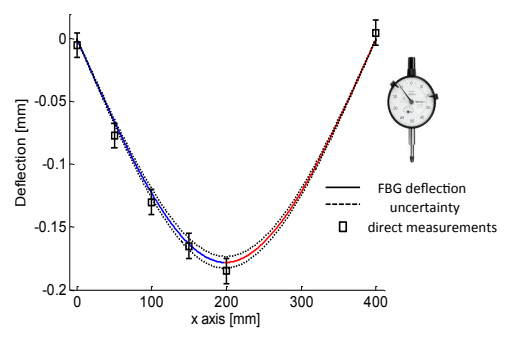

Figure 3: a) Strain measurements vs the acquisition time and as function of the applied mass (W0, W1, W2, W3 and W4); b) Strain profile for maximum weight applied in the middle, the dashed lines represents the uncertainty obtained moving the measurements of $\pm 1 \mu \varepsilon$; c) Deformation profile for maximum weight applied in the middle.

transfer operation. Figure $3 \mathrm{~b}$ shows the strain profile along the $\mathrm{x}$ axis when the $\mathrm{W}_{4}$ mass is placed in the middle of the panel. A liner shape of the strain is calculated imposing that the strain (that is proportional to the torque) is null in the position of the holders. Applying the relation described in 2.2 the deformation profile shown in figure $3 \mathrm{c}$ is obtained. Finally, in order to validate the proposed approach, the obtained deformation is also compared with measurements performed with a mechanical comparator (with resolution of $\pm 10 \mu \mathrm{m}$ ). The data exhibit a very good agreement. Maximum deflection of $178 \pm 5 \mu \mathrm{m}$ in the middle of the panel was estimated by FBGs whereas the direct measurement reveal a maximum deformation of $185 \pm 10 \mu \mathrm{m}$.

\section{Conclusion}

In this paper we present a preliminary analysis on the use of FBG sensors integrated with tracking particle detector support panel as real-time deflection monitoring system. The results prove that the proposed approach has the potentialities to ensure a continue monitoring of the deformation and bending of a typical support panel for Micro Pattern Gaseous Detectors. The achieved resolution of this method is of the order of tens of microns, that is adequate to the desired requirements. Further works will be addressed to implement the $2 \mathrm{D}$ analysis of the support panel. To this purpose numerical mechanical-analysis of the final detector will be used to reveal the number and optimized position of the FBG sensors to determinate all possible stretching and bending of the detector due to mechanical stress and undesired thermal change.

\section{References}

[1] A. Iadicicco, A. Cutolo, A. Cusano, "Fiber Bragg Grating Sensors - Advancements and Industrial Applications," Advances in Science and Technology Vol. 55, n. 213, 2008

[2] G. Berruti, M. Consales, M. Giordano, L. Sansone, P. Petagna, S. Buontempo, G. Breglio, A. Cusano, "Radiation hard humidity sensors for high energy physics applications using polyimide- coated fiber Bragg gratings sensors," Sensors and Actuators B Vol. 177, pp. 94-102, 2013

[3] E. Carrera, G. Giunta, M. Petrolo, Beam Structures: Classical and Advanced Theories, John Wiley \& Sons, 2011 